\title{
Acupuncture at a Single Acupoint, BL1 (Jingming), Compared to Artificial Tears in Moderate to Severe Dry Eye Disease: A Randomized Controlled Trial
}

xue zhang ( $\sim$ zhangxueresume@aliyun.com )

South Area of Guang'anmen Hospital https://orcid.org/0000-0002-8812-1717

\section{Bo Zhang}

South area of Guang'anmen Hospital

\section{Siyang Peng}

Guang'anmen hospital

\section{Guoliang Zhang}

Guang'anmen hospital

\section{Jumei Ma}

South area of Guang'anmen hospital

\section{Wenzeng Zhu}

Guang'anmen hospital

\section{Research Article}

Keywords: dry eye, acupuncture, RCT, Artificial Tears, SIT

Posted Date: October 18th, 2021

DOl: https://doi.org/10.21203/rs.3.rs-564123/v1

License: (1) This work is licensed under a Creative Commons Attribution 4.0 International License. Read Full License 


\section{Abstract}

\section{Background}

The global incidence of dry eye disease (DED) is very high. DED seriously affects the quality of life of patients; however, the current curative effect of medicine for moderate to severe DED is poor. This randomized clinical trial was planned to investigate the effect of acupuncture compared with artificial tears on moderate to severe DED.

\section{Methods}

A randomized clinical trial was performed at 2 hospitals in China. 120 DED patients were randomly equally divided into an acupuncture and an artificial tear group. Either acupuncture or artificial tears was performed for an 8-week period, and a 32-week follow-up was performed. The primary outcome measure was the Schirmer-I test (SIT) value. The secondary outcome measures included the numerical rating scale (NRS) for improvement in ocular symptoms, the ocular surface disease index (OSDI), the tear-film breakup time (TBUT), corneal fluorescein staining (CFS), and acupuncture acceptability. Adverse events also were monitored and documented.

Results

For the primary outcome, the mean changes in the SIT values were significantly different between the acupuncture $(5.75[2.53-9.75])$ and artificial tear $(0.52[-1.18-2.46])$ groups at week $8(P=0.01)$. The OSDI decreased by $-16.14(-35.40-16.30)$ and $-7.65(-19.25-15.05)$ from baseline to week 8 in the acupuncture and artificial tear groups, respectively $(P<0.05)$. A significant decrease was found in the NRS score for eye dryness, eye pain, and blurred vision in the acupuncture group, as compared to the artificial tear group. However, the change in the average symptom NRS score, TBUT, and CFS did not differ significantly at week 8 . Five cases experienced acupuncture-related adverse events.

\section{Conclusions}

This randomized clinical trial found that acupuncture at a single acupoint, BL1, significantly promoted tear secretion and improved multiple eye discomfort symptoms. Acupuncture showed greater benefits than artificial tears for moderate to severe DED. However, the study findings warrant verification.

Trial registration

Registration number: ChiCTR1800015831. Name of trial registry: Efficacy and safety of acupuncture in the treatment of moderate to severe dry eye disease: a randomized controlled trial.

\section{Background}


Dry eye disease (DED) is a chronic ocular surface disease accompanied by symptoms of eye discomfort [1]. It refers to abnormal tear quality or quantity and dynamics, of any cause, resulting in tear-film instability and/or eye surface abnormalities. DED symptoms include eye dryness, foreign body sensation, pain, visual fatigue, and blurred vision. The global DED incidence varies (5-34\%) [2], and is higher in Asian countries. Moreover, the incidence has increased with the recent increased exposure to display screens, abuse of eye medicines, and continuous improvement in DED diagnosis. DED causes include tear-film instability, tear hyperosmolality, ocular surface inflammation, injury, and neurosensory abnormalities [3]. It increases financial burden, and reduces work efficiency, and quality of life [4-6].

Based on expert consensus [7], Artificial tears (ATs) are among the most commonly used drug treatments; ATs lubricate the eye surface, supplement tears, and reduce tear osmotic pressure, but only temporarily relieve DED symptoms. Effective treatment for severe DED is currently lacking. Non-drug treatments include surgery, lacrimal duct embolism, psychological counseling, physiotherapy, health guidance, wet chamber mirror, and silicone eye masks. Surgical treatment may have risks and side-effects, making it a less acceptable treatment strategy. The efficacy of other therapies is not established; thus, these are not widely used [8].

Acupuncture therapy has long history of use in China. Systematic reviews of randomized controlled trials (RCTs) have demonstrated that acupuncture is effective for DED [9-11]. However, previous studies [12, 13] showed that acupuncture treatment only improved subjective DED, but did not significantly increase Schirmer-I test (SIT) scores compared to AT. Moreover, previous clinical studies of acupuncture generally included participants with mild to moderate disease, and the efficacy of acupuncture on moderate to severe DED remains uncertain [14]. Furthermore, the effect of only using periocular acupoints for treated DED was not significant based on a systematic review [15]. Thus, further studies with periocular acupoints accompanied by effective acupuncture manipulate are needed to investigate the effects for patients with moderate to severe DED. Although ATs are currently recommended as a conservative and effective treatment, they cannot replace naturally secreted tears as they lack the oxygen, vitamin A, mucoprotein, and other nutrients present in naturally secreted tears. In our previous clinical study [16], only acupuncture at the BL1 (Jingming) was effective in promoting tear secretion and improving DED symptoms.

In this RCT, we compared the effectiveness of acupuncture at BL1, with repeated needle manipulation until tear flow, with ATs for treating moderate to severe DED. We evaluated the improvements in objective and subjective indicators after an 8-week treatment period and a subsequent 24-week follow-up period.

\section{Methods}

This 2-center, RCT was approved by the Ethics Committee of Guang'anmen Hospital of China Academy of Chinese Medical Sciences. The study commenced on September 1, 2018 and was completed on December 31, 2020. The flow chart is shown in Fig. 1. Before inclusion, each participant provided written informed consent. The study protocol has been published previously [17]. The original trial protocol and 
statistical analysis methods are available (Supplementary 1 ). The trial was conducted and reported in accordance with CONSORT guidelines.

\section{Study design}

Patients were randomized to the acupuncture or AT groups based on a random distribution sequence. Clinical Laboratory of affiliated drugs of Guang'anmen Hospital was responsible for random grouping by computer. The grouping results were placed in 120 opaque sealed envelopes, which were serially numbered and kept by a researcher not involved in treatment procedures or data analysis. After informed consent was obtained, the researcher opened the envelope according to the order in which the participant entered the trial and was provided for the prescribed treatment. All participants received either acupuncture or ATs 3 times per week over the 8-week period, and both groups were followed-up for another 24 weeks.

\section{Study population}

DED participants were recruited from advertisements posted by the hospital. Participants were eligible only they were 18-60 years old and met the diagnostic criteria of moderate-to-severe DED $[18,19]$ : They were required to have one or more symptoms of DED (e.g. eye dryness, foreign body sensation, itching, burning, stinging, visual fatigue, photophobia or blurred vision ) with a severity numerical rating scale (NRS) of $\geq 4$ (range: $0-10$ ). a Schirmer-I test (SIT) (with the application of cocaine, a local anesthetic) value $<10 \mathrm{~mm} / 5$ minutes, a tear film break-up time (TBUT) $<10$ seconds, and corneal fluorescein staining (CFS) scale $\geq 1$. Only the more severely affected eye was analyzed. Major exclusion criteria were a DED surgery history within 3 months, severe ocular diseases or immune system diseases, current use of contact lenses, and pregnancy or lactation. Participants were permitted to continue their other chronic treatments.

\section{Intervention}

Treatments were initiated after randomization immediately. Health education was provided for all participants before treatment, and patients were asked to reduce their exposure to mobile phones and ensure that they slept sufficiently.

The treatment plan was derived from the consensus of acupuncturists and ophthalmologists based on a pilot trial. Acupuncture was administered at BL1 in the acupuncture treatment group. BL1 is known as the tear hole acupoint, and is associated with tear secretion. It is commonly used for treating eye diseases. Huatuo stainless-steel needles $(0.3 \times 100 \mathrm{~mm}$, Suzhou Medical Appliance Factory, Suzhou, China) were used in the acupuncture group, in accordance with the STRICTA guidelines. Acupuncturists had at least 5 years' acupuncture experience and received training before administering treatments in this study. The training included a detailing how to perform the acupuncture. The BL1 acupoint is located slightly above the inner canthus. (Fig. 1) While the participant sat on a chair, the acupuncturist lifted the upper eyelid of the subject with the left hand, fully exposing the inner canthus of the eye, and punctured the acupoint with the needle, vertically, from the inner canthus of the eyes at approximately 0.5 inches. The needle was 
then removed and re-inserted vertically at this position, approximately every 3 seconds. After a number of insertions, tears flowed freely from the eye, at which point the acupuncturist removed the needle.

Treatment was administered 3 times per week for 8 weeks.

The control group was treated with sodium hyaluronate eyedrops (EUSAN GmbH, Saarbrücken, Germany). Subjects were asked to use them as needed ( 1 drop, 4 times per day, in the early morning, early afternoon, afternoon, and before bedtime), with an average interval of 6 hours, for 8 weeks consecutively. A diary recording the frequency of ATs used during the treatment period was collected at each visit.

\section{Outcome measurements}

\section{Primary outcome measure}

The primary outcome was the increase from baseline in the value of the SIT, with anesthesia, at week 8. The SIT was performed using a tear secretion test paper (Color Bar ${ }^{\mathrm{TM}}$ Schirmer tear test, Eagle Vision, Inc., Memphis, TN, USA). Tear volume was measured by placing the strip down the outer third of the eyelid and measuring the length of the wet part of the test strip 5 minutes later (the SIT value). Although there is no clinical significance yet, the SIT value is considered as an important indicator of DED severity and positively correlates with DED symptoms and quality of life [20].

\section{Secondary outcome measures}

Secondary outcomes included the following. The SIT value with anesthesia at baseline, week 4, and week 32 was measured, similarly to week 8 .

The average DED symptom intensity of past week, as assessed by the participant with a selfadministered numerical rating scale (NRS) (range: 0-10), was based on well-known DED symptoms (eye dryness, foreign body sensation, itching, burning, stinging, visual fatigue, photophobia, blurred vision) at baseline, weeks 4,8 , and 32 . The individual score and the mean of these 8 symptoms scores (global symptom score) were calculated and compared.

The Ocular Surface Disease Index (OSDI) was assessed at baseline, weeks 4, 8, and 32. The OSDI is commonly used to evaluate the influence of ocular surface disease on patients' daily life. The scale includes 3 aspects: eye symptoms, visual-related function, and environmental stimulus factors; the higher the OSDI score (range: 0-100), the more serious the impact of the disease on daily life. The change in the total scores reflected the overall improvement in the patients' eye symptoms.

The tear break-up time (TBUT) was measured at baseline, weeks 4, 8, and 32. The TBUT test is used to assess tear-film stability and reflects different pathophysiologies according to rupture mode [21]. After staining with sodium fluorescein $(2.5 \%)$, the time taken for the first dry spot on the tear-film was measured thrice with a slit lamp, and the average time was taken as the TBUT.

Corneal fluorescein staining (CFS), which was used to assess ocular surface damage as well as monitor the clinical response to therapy, was measured at baseline, weeks 4,8 , and 32 . Corneal staining was 
examined under standard illumination with a slit lamp, using a cobalt blue filter, and was graded using the modified Oxford scale [22].

Acceptability of acupuncture or AT was evaluated at week 8, using a 4-point scale, with 1 representing "very difficult to accept," 2 representing "difficult to accept," 3 representing "easy to accept, "and 4 representing "very easy to accept." [23]

All participants were requested to report Adverse events (AEs) in the study during the treatment and follow-up periods. The severity (divided into mild, moderate, severe) of AEs was evaluated by the investigator.

\section{Calculation of sample size and statistical analyses}

The sample size was calculated based on the on the mean SIT value. According to our pilot trial [16], the increase in the mean SIT value in the acupuncture group at week 8 was $3.41 \pm 3.04$. A previous report indicated a mean increase in the SIT value in an artificial tear group of $1.8 \pm 1.6$ [24]. Based on these values, a sample size of 120 was need to detect a difference of 1.61 between two groups, which provided $90 \%$ power with a two-sided $5 \%$ level of significance, considering a $20 \%$ dropout rate.

SPSS Version 26.0 software was used for statistical analysis. All statistical analyses used bilateral tests. $P$ values less than 0.05 were considered to be statistically significant. The analysis of efficacy was based on the principle of intentionality analysis. All participants who had at least one efficacy assessment were all included in the efficacy analysis. The missing data was replaced by using the last observation carried forward method [25].

For data with a natural or adjusted normal distribution, we used mean and $95 \%$ confidence interval (Cls). For data with a non-normal distribution, we used the median and $95 \% \mathrm{Cl}$. Group $t$-tests or non-parametric rank and sum tests were used for between-group comparisons. For within-group comparisons to baseline values, $t$-tests or non-parametric rank and sum tests were used. Independent sample $t$-tests were used to compare different time-points. Categorical variables were described using composition ratios and/or 95\% confidence intervals. Chi-square test or Fisher's exact test was used for comparisons between groups.

\section{Results}

\section{Enrollment}

We screened 167 participants with DED for eligibility, and excluded 47; thus, 120 participants were assigned to either the acupuncture group $(n=60)$ or the AT group $(n=60)$, at a ratio of 1:1. Among them, 113(94.2\%) participants completed at least 4-week treatments and 110(91.7\%) participants completed all the trail (Fig. 2). Baseline characteristics are shown in Table 1; these were similar between the groups. 
Participant Baseline Characteristicsa,b

\begin{tabular}{|c|c|c|}
\hline Characteristic & $\begin{array}{l}\text { Acupuncture } \\
\text { group }(n=60)\end{array}$ & $\begin{array}{l}\text { Artificial Tear } \\
\text { group }(n=60)\end{array}$ \\
\hline Age (y), (mean, 95\%Cl) & $44.16(35.54$ to 49.43$)$ & $41.28(31.78$ to 45.78$)$ \\
\hline Sex, M/F (\%) & $22 / 38$ & $29 / 31$ \\
\hline \multicolumn{3}{|l|}{ Educational level (No. [\%]) } \\
\hline Primary education or less & $16(26.7)$ & 11(18.3) \\
\hline Secondary education & $38(63.3)$ & $42(70.0)$ \\
\hline Tertiary education & $6(10.0)$ & $7(11.7)$ \\
\hline DED duration, median(IQR), mo & $26(6-60)$ & $22(5-60)$ \\
\hline \multicolumn{3}{|l|}{ Profession (№. [\%]) } \\
\hline office worker & $30(50.0)$ & $34(56.7)$ \\
\hline Physical work & $12(20.0)$ & 11(18.5) \\
\hline Retired or unemployed & 18(30.0) & $15(25.0)$ \\
\hline \multicolumn{3}{|l|}{ Comorbidities (No. [\%]) ${ }^{c}$} \\
\hline Cardio vascular and cerebro vascular diseases & $6(10.0)$ & $4(6.7)$ \\
\hline Metabolic disorders & $5(8.3)$ & $6(10.0)$ \\
\hline Eye Conditions & $9(15.0)$ & $7(11.7)$ \\
\hline Other & $2(3.3)$ & $3(5.0)$ \\
\hline Sleep disorders (No. [\%]) & $3(5.0)$ & $3(5.0)$ \\
\hline SIT & $4.37(3.37$ to 5.25$)$ & $3.82(2.84$ to 4.86$)$ \\
\hline Average symptom NRS,(mean, 95\%Cl) & $7.14(4.29$ to 8.85$)$ & $6.83(4.27$ to 8.63$)$ \\
\hline OSDI, (mean, 95\%Cl) & $34.50(29.15$ to 41.23$)$ & $32.20(27.80$ to 40.12$)$ \\
\hline TBUT, (mean, 95\%Cl) & $4.31(3.38$ to 5.25$)$ & $4.40(3.56$ to 5.32$)$ \\
\hline \multicolumn{3}{|c|}{$\begin{array}{l}\text { a SD = standard deviation; DED = dry eye disease; SIT = Schirmer-I test; NRS: numerical rating scale; } \\
\text { OSDI: ocular surface disease index; TBUT: tear break-up time; CFS: corneal fluorescence staining, IQR } \\
\text { = inter-quartile range. }\end{array}$} \\
\hline \multicolumn{3}{|c|}{ b There were no significant between-group differences at baseline. } \\
\hline
\end{tabular}




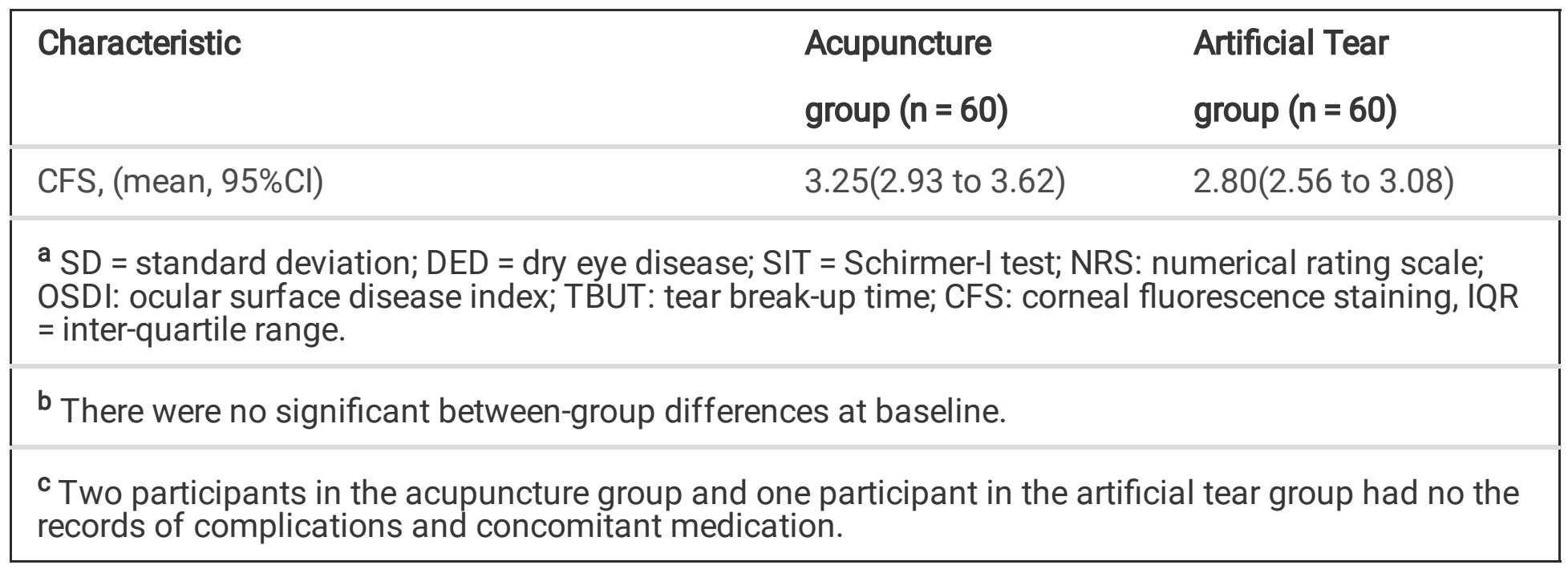

\section{Missing data and dropouts}

Adherence to acupuncture treatments ( 3 times per week for 8 weeks) or AT treatments ( 4 times per day for 8 weeks) was similar in both groups, $90.0 \%$ and $96.7 \%$ respectively. Of the 120 patients, 113 patients completed 4-week treatments and 110 patients completed all treatments and follow-ups. The data of the 120 participants was analyzed in the full analysis set based on intentionality analysis principle. A sensitivity analysis was performed in the per protocol set, which included the participants that completed all the treatments and follow-ups. The original results in this study were stable and reliable based on the sensitivity analysis.

\section{Outcomes}

\section{Primary outcome measures}

For the primary outcome (Table 2), the mean SIT value was $4.37(95 \% \mathrm{Cl}, 3.37-5.25)$ at baseline and 9.25 $(95 \% \mathrm{Cl}, 6.00-12.75)$ at week 8 in the acupuncture group, and $3.82(95 \% \mathrm{Cl}, 2.84-4.86)$ at baseline and $4.34(95 \% \mathrm{Cl}, 2.94-5.98)$ at week 8 in the AT group. The increase in the SIT value at week 8 was greater in the acupuncture group (mean, 5.75) than in the AT group (mean, 0.52), with a mean difference of 5.23 $(95 \% \mathrm{Cl}, 1.34-9.12 ; \mathrm{P}=0.01)$.

Similar results were observed at week 4 (Table 2). The increase in the SIT value at week 4 was greater in the acupuncture group (mean, 5.16) than in the AT group (mean, -0.3) with a mean difference of 5.46 $(95 \% \mathrm{Cl}, 2.73-8.18 ; \mathrm{P}<0.001)$. However, the SIT values were not significantly different between both groups at week 32 . 
TABLE 2. Primary and Secondary Outcomes ${ }^{a}$

\begin{tabular}{|c|c|c|c|c|}
\hline Variable & $\begin{array}{l}\text { Acupuncture } \\
\text { group }(n=60)\end{array}$ & $\begin{array}{l}\text { Artificial Tear } \\
\text { group }(n=60)\end{array}$ & $\begin{array}{l}\text { Difference } \\
(95 \% \mathrm{Cl})\end{array}$ & $P$ value \\
\hline $\begin{array}{l}\text { Primary } \\
\text { outcome }\end{array}$ & $\begin{array}{l}9.25(6.00 \text { to } \\
12.75)\end{array}$ & $\begin{array}{l}4.34(2.94 \text { to } \\
5.98)\end{array}$ & & \\
\hline $\begin{array}{l}\text { SIT at weeks } \\
8, \text { mean }(95 \% \\
\text { CI) }\end{array}$ & 5.75 (2.53 to 9.75$)$ & $\begin{array}{l}0.52(-1.18 \text { to } \\
2.46)\end{array}$ & $5.23(1.34$ to 9.12$)$ & 0.01 \\
\hline \multicolumn{5}{|l|}{$\begin{array}{l}\text { SIT Change, } \\
\text { at weeks } 8, \\
\text { mean }(95 \% \mathrm{Cl})\end{array}$} \\
\hline \multirow{2}{*}{$\begin{array}{l}\text { Secondary } \\
\text { Outcomes }\end{array}$} & \multirow{2}{*}{00000000000000} & 00000000000000 & 00000000000000 & 00000000000000 \\
\hline & & 00000000000000 & 00000000000000 & 00000000000000 \\
\hline \multirow{2}{*}{$\begin{array}{l}\text { Change, SIT, } \\
\text { mean (95\% } \\
\text { CI) } \\
\text { at week 4, } \\
\text { at week 32, }\end{array}$} & $\begin{array}{l}00000000000000 \\
5.16(2.94 \text { to } 7.87)\end{array}$ & $\begin{array}{l}-0.30(-1.62 \text { to } \\
1.28)\end{array}$ & \multirow{2}{*}{$\begin{array}{l}5.46(2.73 \text { to } 8.18) \\
1.89(0.54 \text { to } \\
4.07)\end{array}$} & \multirow{2}{*}{$\begin{array}{l}<0.001 \\
0.066\end{array}$} \\
\hline & 2.05 (0.94 to 4.47$)$ & $\begin{array}{l}0.16(-1.94 \text { to } \\
2.83)\end{array}$ & & \\
\hline \multirow{3}{*}{$\begin{array}{l}\text { Change, } \\
\text { Average } \\
\text { symptom } \\
\text { NRS, b } \\
\text { mean (95\% } \\
\text { CI) }\end{array}$} & \multirow{3}{*}{00000000000000} & 00000000000000 & 00000000000000 & 0000000000 \\
\hline & & \multirow[t]{2}{*}{00000000000000} & \multirow[t]{2}{*}{00000000000000} & \multirow[t]{2}{*}{000000000000000} \\
\hline & & & & \\
\hline at week 4, & $\begin{array}{l}-2.26(-5.07 \\
\text { to } 1.63)\end{array}$ & $\begin{array}{l}-1.69(-5.05 \text { to } \\
1.89)\end{array}$ & $\begin{array}{l}0.57(-2.15 \text { to } \\
2.46)\end{array}$ & 0.162 \\
\hline at week 8 & $\begin{array}{l}-2.96(-4.94 \text { to } \\
1.87)\end{array}$ & $\begin{array}{l}-2.55(-4.25 \text { to } \\
1.95)\end{array}$ & $\begin{array}{l}0.38(-2.46 \text { to } \\
3.08)\end{array}$ & 0.084 \\
\hline at week 32, & $\begin{array}{l}-3.10(-2.46 \\
\text { to } 1.54)\end{array}$ & $\begin{array}{l}-1.22(-0.89 \text { to } \\
0.09)\end{array}$ & $\begin{array}{l}1.88(-0.46 \text { to } \\
3.57)\end{array}$ & 0.036 \\
\hline \multirow{2}{*}{$\begin{array}{l}\text { Change, OSDI, } \\
\text { mean }(95 \% \\
\text { CI) }\end{array}$} & 00000000000000 & 00000000000000 & 00000000000000 & 00000000000000 \\
\hline & $\begin{array}{l}-9.46(-32.66 \text { to } \\
13.59)\end{array}$ & $\begin{array}{l}-5.26(-20.05 \text { to } \\
21.89)\end{array}$ & $\begin{array}{l}4.20(-14.35 \text { to } \\
18.56)\end{array}$ & \multirow{2}{*}{$\begin{array}{l}0.140 \\
0.036\end{array}$} \\
\hline $\begin{array}{l}\text { at week } 4 \\
\text { at week } 8\end{array}$ & $\begin{array}{l}-16.14(-35.40 \text { to } \\
16.30)\end{array}$ & $\begin{array}{l}-7.65(-19.25 \text { to } \\
15.05)\end{array}$ & $\begin{array}{l}8.49(-5.26 \text { to } \\
22.14)\end{array}$ & \\
\hline at week $32^{b}$ & $\begin{array}{l}-18.18(-26.46 \text { to } \\
16.54)\end{array}$ & $\begin{array}{l}-6.03(-21.89 \text { to } \\
19.32)\end{array}$ & $\begin{array}{l}12.15(-4.68 \text { to } \\
15.46)\end{array}$ & \\
\hline
\end{tabular}

Change, 
TBUT, mean

(95\% Cl)

\begin{tabular}{|c|c|c|c|c|}
\hline at week 4 & $1.56(-0.13$ to 3.06$)$ & $\begin{array}{l}0.16(-0.80 \text { to } \\
1.16)\end{array}$ & $\begin{array}{l}1.40(-0.46 \text { to } \\
3.27)\end{array}$ & 0.136 \\
\hline at week 8 & $1.72(-.12$ to 3.25$)$ & $\begin{array}{l}0.44(-0.76 \text { to } \\
1.68)\end{array}$ & $\begin{array}{l}1.28(-1.01 \text { to } \\
3.26)\end{array}$ & 0.294 \\
\hline at week 32, & $0.46(-0.14$ to 2.46$)$ & $\begin{array}{l}0.12(-0.43 \text { to } \\
1.89)\end{array}$ & $\begin{array}{l}0.34(-1.01 \text { to } \\
2.26)\end{array}$ & 0.569 \\
\hline $\begin{array}{l}\text { Change, CFS, } \\
\text { mean }(95 \% \\
\text { CI) }\end{array}$ & & & 00000000000000 & 00000000000000 \\
\hline at week 4 & $-2.99(1.00$ to 4.25$)$ & $\begin{array}{l}-2.38 \text { (0.40 to } \\
3.96)\end{array}$ & $\begin{array}{l}0.61(-0.18 \text { to } \\
2.54)\end{array}$ & 0.136 \\
\hline at week 8 & $-3.25(0.98$ to 4.58$)$ & $\begin{array}{l}-3.06 \\
4.14)\end{array}$ & $0.29(-0.85$ to & 0.214 \\
\hline at week 32, & $-2.15(1.54$ to 2.46$)$ & $\begin{array}{l}-2.57(-0.09 \text { to } \\
0.89)\end{array}$ & $\begin{array}{l}0.14(-1.07 \text { to } \\
1.92)\end{array}$ & 0.325 \\
\hline $\begin{array}{l}\text { Treatment } \\
\text { acceptability }\end{array}$ & $\begin{array}{l}\text { Little difficult: } \\
6(10 \%) \text {, }\end{array}$ & $\begin{array}{l}\text { Little difficult: } \\
5(8.3 \%)\end{array}$ & & 0.130 \\
\hline \multirow[t]{3}{*}{$\begin{array}{l}\text { assessment, } \\
\text { number }(\%)^{\mathbf{d}}\end{array}$} & $\begin{array}{l}\text { Moderate: } 16 \\
(26.7 \%)\end{array}$ & $\begin{array}{l}\text { Moderate: } 10 \\
(16.7 \%) \text {, }\end{array}$ & & \\
\hline & $\begin{array}{l}\text { Easy: } 32 \\
(53.3 \%),\end{array}$ & $\begin{array}{l}\text { Easy: } \\
35(58.3 \%),\end{array}$ & & \\
\hline & $\begin{array}{l}\text { Very easy: } 6 \\
(10.0 \%)\end{array}$ & $\begin{array}{l}\text { Very easy: } \\
10(16.7 \%)\end{array}$ & & \\
\hline
\end{tabular}

a SIT = Schirmer-I test; NRS: numerical rating scale; OSDI: ocular surface disease index; TBUT: tear breakup time; CFS: corneal fluorescein staining; $\mathrm{Cl}$ : confidence intervals.

b Scoring is the sum of the totals divided by 8 (8 symptoms in total).

${ }^{c}$ Scoring was additive (0 [better outcomes] to 44 [worse outcomes]). Minimal clinically important difference: 13.9 .

d Treatment acceptability assessed by the chi-square tests. All participants (120) were included in acceptability

assessment and the participants who did not completed the treatments were all evaluated as a little difficulty of acceptability.

\section{Secondary outcome measures}


No significant differences in the change in mean DED symptom NRS scores were found between the groups (all $P>0.05$ ). Symptoms varied among participants, and not all participants experience every listed symptom. The acupuncture group had significantly decreased NRS scores for eye dryness, eye pain, and blurred vision compared to the AT group at multiple time-points. There was no significant difference between both groups for other symptoms.

The acupuncture group showed a more significant decrease than the AT group in the OSDI score at weeks $8(8.49[95 \% \mathrm{Cl},-5.26-22.14])$ and $32(12.15[95 \% \mathrm{Cl},-4.68-15.46])($ all $\mathrm{P}<0.05)$, but not at week $4(\mathrm{P}>$ $0.05)$.

There were no significant differences in the change in TBUT between both groups. A minor difference of $1.4(95 \% \mathrm{Cl},-0.46-3.27)$ was detected at week $4,1.28(95 \% \mathrm{Cl},-1.01-3.26)$ at week 8 , and $0.34(95 \% \mathrm{Cl}$, $-1.01-2.26$ ) at week 32. The differences between both groups were not significant (all $P>0.05$ ).

There were no significant differences in the change in CFS from baseline to all time-points between both groups. The reduction in CFS was similar between both groups.

Acupuncture and AT acceptability were assessed after 8 weeks of intervention. $63.3 \%$ of participants in the acupuncture group and $75.0 \%$ in the AT group is easy acceptable or very easy acceptable. No significant differences were observed between the groups $(P=0.13)$. The detailed data are shown in Table 2.

\section{Safety Outcomes}

Treatment-related AEs occurred in $8.3 \%$ of participants in the acupuncture group; AEs included mild bleeding, which was absorbed quickly, or moderate bleeding, which was resolved by cold compress. The incidence of AEs in the AT group was $3.4 \%$, which included nausea (1 participant) and dizziness (1 participant). However, it was unclear whether these AEs were related to the treatment. No other AEs occurred. (Table 3 ) 
Table 3

Adverse Events.

\begin{tabular}{|lll|}
\hline Adverse event & $\begin{array}{l}\text { Participants (No. [\%]) } \\
\text { Acupuncture } \\
\text { group }(\mathbf{n}=60)\end{array}$ & $\begin{array}{l}\text { Artificial Tear } \\
\text { group }(\mathbf{n}=60)\end{array}$ \\
\hline Overal & $5(8.3)$ & $2(3.4)$ \\
Bleeding(mild) & $3(5)$ & 0 \\
Bleeding(moderate) & $1(1.7)$ & 0 \\
Sharp pain & $1(1.7)$ & 0 \\
Feel nauseated & 0 & $1(1.7)$ \\
Dizziness & 0 & $1(1.7)$ \\
\hline $\begin{array}{l}\text { Adverse events were analyzed in all participants who received treatment. Adverse events were } \\
\text { counted by type other than frequency in the same participant. Adverse events with different types } \\
\text { occurring in a single participant were defined as independent adverse events. An adverse event with } \\
\text { multiple occurrences in a single participant was defined as 1 adverse event. }\end{array}$ \\
\hline
\end{tabular}

\section{Discussion}

In this RCT, we compared acupuncture and ATs for DED. After 4 and 8 weeks of treatment, acupuncture resulted in greater improvement in the SIT value than AT. The overall incidence of AEs was low.

The SIT value provides direct objective evidence for the therapeutic value of acupuncture. In this study, the SIT value in the acupuncture group increased by 5.16 and 5.75 after 4 and 8 weeks, respectively. Previous studies showed an increase of 0.41 [12] and 0.96 [13], which was significantly lower than that in our study. The acupuncture method used in this study may have been more conducive to promoting tear secretion than those used in previous studies, due to the acupoint used, the stronger and longer duration of needling manipulation, and tear-flow method after needling manipulation.

The OSDI is also an important indicator of DED symptoms. In this trial, the change in the OSDI score in the acupuncture group (Table 2) was consistent with previous findings. In our study, the OSDI score decreased by 16.14 after 8 weeks in the acupuncture group, similar to the decrease of 16.11 found previously [12]. A median reduction of 13.9 in the OSDI score represents the minimal clinically important difference [26].

Although previous studies have shown that acupuncture can improve DED symptoms, there were some differences in findings. In our study, acupuncture significantly improved the symptoms of dry eye, itching, eye pain, and blurred vision; however, it was less effective in improving other DED symptoms (photophobia, blurred vision, visual fatigue and foreign body sensation) than AT, possibly due to the acupuncture point used, needle manipulation, and inclusion of moderate to severe DED participants. In 
this study, acupuncture stimulated lacrimal secretion and improved lacrimal gland function, rapidly relieving many DED symptoms in a sustained manner. A previous study showed that acupuncture could release opioid peptides, which may explain the relief of eye pain by acupuncture [27]. Acupuncture also altered the tear composition and regulated the immune system, thereby reducing inflammation or an allergic reaction at the eye surface [28].

In this study, objective indicators other than the SIT value did not improve significantly. A previous study on DED showed that the TBUT increased significantly, by 0.95 , after acupuncture, but not after sham acupuncture [29]. Another study showed that TBUT increased by 1.93 after acupuncture treatment [13].

Participants in both groups generally found their treatment acceptable. The use of a single acupoint, rather than multiple acupoints as in previous studies, reduced the pain of acupuncture, possibly contributing to its acceptability.

Most previous studies showed that acupuncture treatment only improved subjective DED assessments, but did not investigate objective assessments or did not find significant improvement in the objective indicators (SIT, TBUT, CFS) [30, 31]. In this study, acupuncture treatment at BL1 with needle manipulation resulted in approximately 10 times more tear secretion than in the AT group, suggesting the mechanism underlying the efficacy of acupuncture. The BL1 point is located on the lacrimal caruncle of the inner canthus, and the tissues in the lacrimal caruncle and Krause glands may come in contact during acupuncture, which could regulate basic tear secretion and help maintain ocular surface homeostasis. When acupuncture was performed in the nasal direction, the sensory neurons in the lacrimal reflex arc may have been stimulated, thus triggering reflexes through the trigeminal nerve, which were transmitted to the lacrimal gland to promote tear production and regulate tear secretion [32].

The anatomical position of BL1 may be related to the better curative effect observed in many previous acupuncture trials for ophthalmic diseases [33]. Modern neuroanatomy indicates that the BL1 point belongs to the trigeminal nerve distribution. When the BL1 point is stimulated, the trigeminal nerve could produce excitatory signals in the brain, which could improve visual function.

This study had some limitations. First, we did not limit the subtype of DED when including study participants. Our findings may be more relevant to the lacrimal deficiency type. Second, because the AT group was the control group, blinding was not performed, which may have affected study results. Thirdly, the effect of acupuncture may be related to the psychological expectation of the subjects before treatment; this should be investigated in future.

\section{Conclusions}

Acupuncture at the BL1 point alone can significantly improve lacrimal secretion and symptoms of moderate and severe DED, with a better curative effect than that of AT. Acupuncture treatment was acceptable to patients and there were no serious AEs. Thus, this simple acupuncture treatment could be considered as a safe, effective, and acceptable for moderate to severe DED. 


\section{Abbreviations}

DED = dry eye disease, AT = artificial tear, SIT = Schirmer-I test, NRS = numerical rating scale, OSDI = ocular surface disease index, TBUT = tear break-up time, CFS = corneal fluorescein staining, RCT = randomized controlled trial, $\mathrm{AEs}=$ adverse events, $\mathrm{Cl}=$ confidence interval, DEWS = Dry Eye Work Shop, ITT = intentionto-treat, SD = standard deviation, SPIRIT = Standard Protocol Items: Recommendations for Interventional Trials, STRICTA = Standards for Reporting Interventions in Clinical Trials of Acupuncture.

\section{Declarations}

\section{Acknowledgements}

The authors acknowledge financial support, and thank the editors of AJE for their help in proofreading the manuscript.

\section{Authors' contributions}

ZWZ had full access to all of the data in the study and takes responsibility for the integrity of the data and the accuracy of the data analysis.

Concept and design: ZWZ, ZX.

Acquisition, analysis, or interpretation of data: ZX, PSY, ZB.

Drafting of the manuscript: ZX.

Critical revision of the manuscript for important intellectual content: ZWZ, ZX.

Statistical analysis: PSY

Obtained funding: ZWZ, ZX.

Administrative, technical, or material support: ZWZ, ZGL, MJM

Supervision: ZX, ZGL, MJM.

All authors approved the final manuscript.

\section{Funding}

This trial is supported by the South Area of Guang'anmen Hospital science Fund (Funding no. Y2017-11) and the Beijing science and Technology Development Fund for Chinese Medicine (Funding no. JJ201857).

\section{Availability of data and materials}


The full data set will be made available. Requests for the data to be released should be sent to ZWZ (principal investigator).

\section{Ethics approval and consent to participate}

Central ethical approval has been confirmed from China Academy of Chinese Medical Sciences (ethical number: 2018-012-KY-01)

\section{Competing interests}

The authors declare no conficts of interest. The lead author confrms that this manuscript is an accurate, honest and transparent account of the study undertaken and reported, with no aspects being omitted and any discrepan- cies explained.

\section{References}

1. The definition and classification of dry eye disease: report of the Definition and Classification Subcommittee of the International Dry Eye WorkShop. (2007). Ocul Surf. 2007;5(2):75-92.

2. Messmer EM. The pathophysiology, diagnosis, and treatment of dry eye disease. Dtsch Arztebl Int. 2015;112(5):71-81. quiz 82.

3. Simsek C, Dogru M, Kojima T, Tsubota K. Current Management and Treatment of Dry Eye Disease. Turk J Ophthalmol. 2018;48(6):309-13.

4. Zeev MS, Miller DD, Latkany R. Diagnosis of dry eye disease and emerging technologies. Clin Ophthalmol. 2014;8:581-90.

5. Dana R, Bradley JL, Guerin A, et al. Estimated Prevalence and Incidence of Dry Eye Disease Based on Coding Analysis of a Large, All-age United States Health Care System. Am J Ophthalmol. 2019;202:47-54.

6. Barabino S, Labetoulle M, Rolando M, Messmer EM. Understanding Symptoms and Quality of Life in Patients With Dry Eye Syndrome. Ocul Surf. 2016;14(3):365-76.

7. Management and therapy of dry eye disease: report of the Management and Therapy Subcommittee of the International Dry Eye WorkShop (2007). Ocul Surf. 2007;5(2):163-178.

8. Shen Lee B, Kabat AG, Bacharach J, Karpecki P, Luchs J. Managing Dry Eye Disease and Facilitating Realistic Patient Expectations: A Review and Appraisal of Current Therapies. Clin Ophthalmol. 2020;14:119-26.

9. Yang L, Yang Z, Yu H, Song $\mathrm{H}$. Acupuncture therapy is more effective than artificial tears for dry eye syndrome: evidence based on a meta-analysis. Evid Based Complement Alternat Med. 2015;2015:143858.

10. Kim BH, Kim MH, Kang SH, Nam HJ. Optimizing acupuncture treatment for dry eye syndrome: a systematic review. BMC Complement Altern Med. 2018;18(1):145. 
11. Lee MS, Shin BC, Choi TY, Ernst E. Acupuncture for treating dry eye: a systematic review. Acta Ophthalmol. 2011;89(2):101-6.

12. Kim TH, Kang JW, Kim KH, et al. Acupuncture for the treatment of dry eye: a multicenter randomised controlled trial with active comparison intervention (artificial teardrops). PLoS One. 2012;7(5):e36638.

13. Liu Q, Liu J, Ren C, et al. Proteomic analysis of tears following acupuncture treatment for menopausal dry eye disease by two-dimensional nano-liquid chromatography coupled with tandem mass spectrometry. Int J Nanomedicine. 2017;12:1663-71.

14. Kuo YK, Lin IC, Chien LN, et al. Dry Eye Disease: A Review of Epidemiology in Taiwan, and its Clinical Treatment and Merits. J Clin Med. 2019;8(8).

15. Wei QB, Ding N, Wang JJ, Wang W, Gao WP. Acupoint selection for the treatment of dry eye: A systematic review and meta-analysis of rand.

16. omized controlled trials. Exp Ther Med. 2020;19(4):2851-60.

17. Zhu WZ, Ni J, et al. A preliminary observational study of 27 cases acupuncture for dry eye disease by Jingming acupuncture point. Chinese Acupuncture Moxibustion. 2017;1:37.

18. Zhang X, Liu Z, Ding W, Zhang J, Shi H, Zhu W. Efficacy and safety of acupuncture at a single BL1 acupoint in the treatment of moderate to severe dry eye disease: Protocol for a randomized, controlled trial. Medicine. 2018;97(22):e10924.

19. Thulasi P, Djalilian AR. Update in current diagnostics and therapeutics of dry eye disease. Ophthalmology. 2017;124:27-s33.

20. Baudouin Christophe,Figueiredo Francisco C, Messmer Elisabeth M, et al. A randomized study of the efficacy and safety of $0.1 \%$ cyclosporine A cationic emulsion in treatment of moderate to severe dry eye.[. J]Eur J Ophthalmol. 2017;27:520-30.

21. Brott NR, Ronquillo Y. Schirmer Test. In: StatPearls. Treasure Island (FL)2020.

22. Eom HD, Jung JU, Lee KP, et al. Simplified Classification of Tear Film Break-Up Patterns and Their Clinicopathological Correlations in Patients With Dry Eye Disease. Eye Contact Lens. 2020.

23. Bron AJ, Evans VE, Smith JA. Grading of corneal and conjunctival staining in the context of other dry eye tests. Cornea. 2003;22(7):640-50.

24. Zhang X, Wang Y, Wang Z, Wang C, Ding W, Liu Z. A Randomized Clinical Trial Comparing the Effectiveness of Electroacupuncture versus Medium-Frequency Electrotherapy for Discogenic Sciatica. Evid Based Complement Alternat Med. 2017;2017:9502718.

25. Robert PY, Cochener B, Amrane M, et al. Efficacy and safety of a cationic emulsion in the treatment of moderate to severe dry eye disease: a randomized controlled study. Eur $\mathrm{J}$ Ophthalmol. 2016;26(6):546-55.

26. Zhao L, Chen J, Li Y, et al. The long-term effect of acupuncture for migraine prophylaxis: a randomized clinical trial. JAMA Intern Med 2017;177:508-15. 10.1001/jamainternmed.2016.9378 28241154. 
27. Miller KL, Walt JG, Mink DR, et al. Minimal clinically important difference for the ocular surface disease index. Arch Ophthalmol. 2010;128(1):94-101.

28. Nepp J, Jandrasits $K$, Schauersberger $J$, et al. Is acupuncture an useful tool for pain-treatment in ophthalmology? Acupunct Electrother Res. 2002;27(3-4):171-82.

29. Shi JL, Miao WH. [Effects of acupuncture on lactoferrin content in tears and tear secretion in patients suffering from dry eyes: a randomized controlled trial]. Zhong Xi Yi Jie He Xue Bao. 2012;10(9):1003-8.

30. Lan W, Tong L. Acupuncture has effect on increasing tear break-up time: acupuncture for treating dry eye, a randomized placebo-controlled trial. Acta Ophthalmol. 2012;90(1):e73.

31. Dhaliwal DK, Zhou S, Samudre SS, Lo NJ, Rhee MK. Acupuncture and dry eye: current perspectives. A double-blinded randomized controlled trial and review of the literature. Clin Ophthalmol. 2019;13:731-40.

32. Jeon JH, Shin MS, Lee MS, et al. Acupuncture reduces symptoms of dry eye syndrome: a preliminary observational study. J Altern Complement Med. 2010;16(12):1291-4.

33. Gong L, Sun X. Treatment of intractable dry eyes: tear secretion increase and morphological changes of the lacrimal gland of rabbit after acupuncture. Acupunct Electrother Res. 2007;32(3-4):223-33.

34. Zhang W, Li H, Zhao N, Hai X, Dong H, Wang J. [Bridge role of Jingming (BL 1) for VDT asthenopia and brainfag based on human instinct]. Zhongguo Zhen Jiu. 2017;37(1):85-7.

\section{Figures}

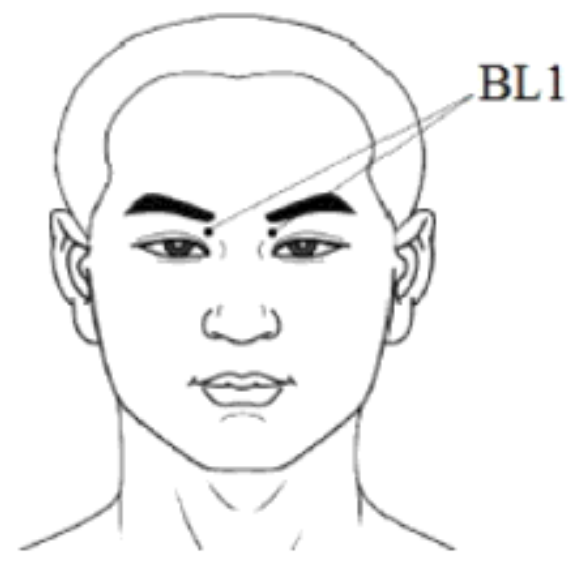

\section{Figure 1}

Location of acupuncture points. 


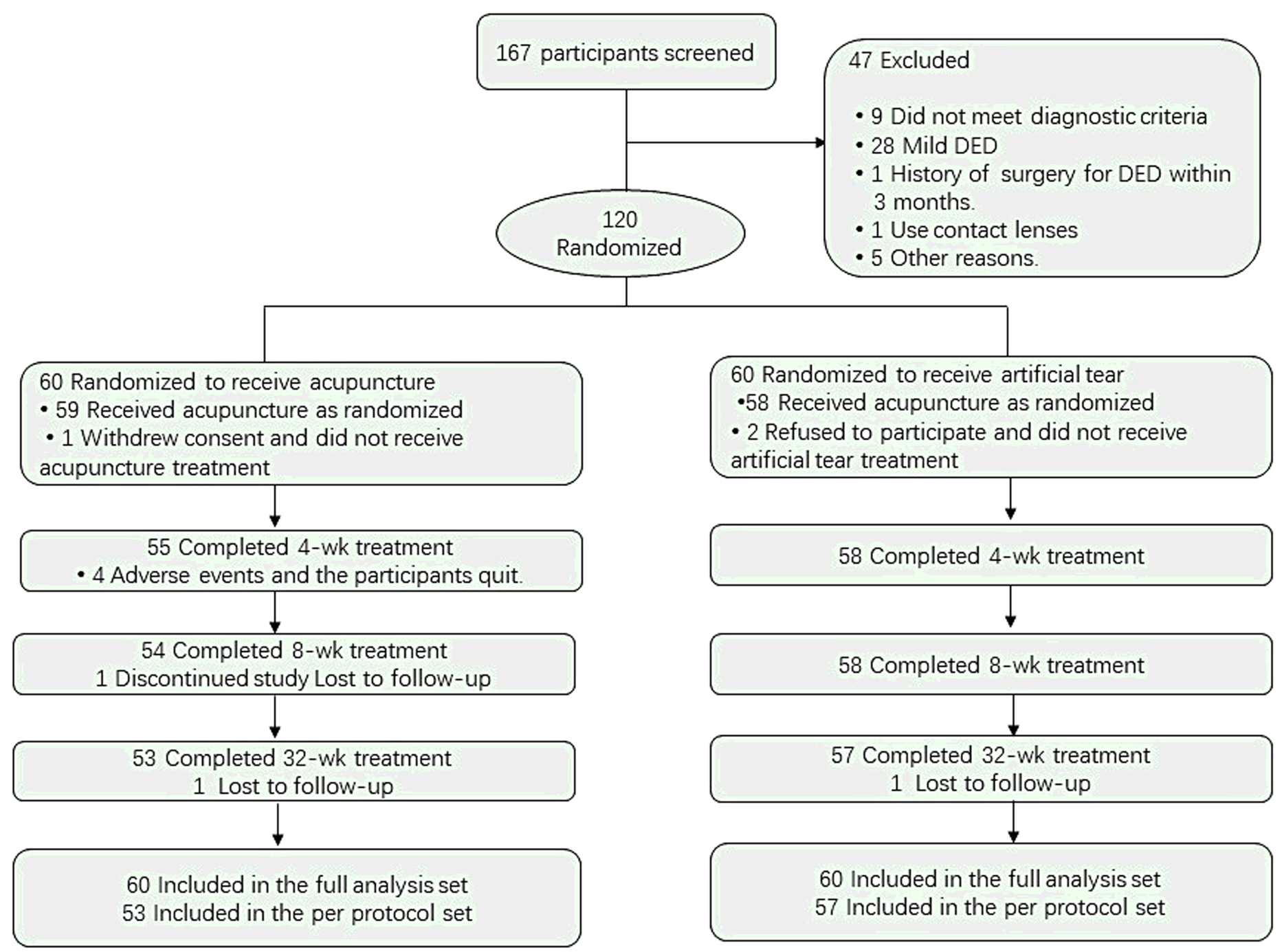

Figure 2

Flow of Participants

\section{Supplementary Files}

This is a list of supplementary files associated with this preprint. Click to download.

- SUPPLEMENT2.CONSORTchecklistofinformation.doc

- SUPPLEMENT1.TrialProtocolandStatisticalAnalysisPlan.pdf 